\section{Vol. 31, Issue 7, July 2008}

Editorials

357 Low Intensity Alternating Electric Fields:

A Potentially Safe and Effective Treatment of

Cancer?

Schroeder, T.; Viglianti, B.L.; Dewhirst, M.W. (Durham, NC)

360 Therapy of Localized Esophageal Cancer:

It Is Time to Reengineer Our Investigative Strategies Ajani, J.A. (Houston, TX)

Original Articles

362 A Pilot Study with Very Low-Intensity, Intermediate-Frequency Electric Fields in Patients with Locally Advanced and/or Metastatic Solid Tumors

Salzberg, M. (Basel); Kirson, E.; Palti, Y. (Haifa); Rochlitz, C. (Basel)

366 Paclitaxel in the Neoadjuvant Treatment for Adenocarcinoma of the Distal Esophagus (AEG I). A Comparison of Two Phase II Trials with Long-Term Follow-Up

Bader, F.G. (Lübeck/Stockholm); Lordick, F. (Heidelberg); Fink, U.; Becker, K.; Höfler, H.; Busch, R. (München); Siewert, J.R.; Ott, K. (Heidelberg)

375 Surgery of Liver Metastasis in Gynecological Cancer - Indication and Results

Kollmar, O.; Moussavian, M.R.; Richter, S.; Bolli, M.; Schilling, M.K. (Homburg)

380 Long-Term Survival Analysis in Metastatic Melanoma: Serum S100B Is an Independent Prognostic Marker and Superior to LDH Egberts, F.; Pollex, A.; Egberts, J.H.; Kaehler, K.C.; Weichenthal, M.; Hauschild, A. (Kiel)

Clinical Cases

387 Bilateral Metastases to Extraocular Muscles from Lobular Breast Carcinoma

Kouvaris, J.R.; Gkongkou, P.V.; Papadimitriou, C.A.;

Papacharalampous, X.N.; Antypas, C.E.; Balafouta, M.J.;

Vlahos, L.J. (Athens)
Band 31, Heft 7, Juli 2008

Editorials

357 Sehr schwache elektrische Felder von intermediärer Frequenz: Eine potentiell sichere und effektive Krebstherapie?

Schroeder, T.; Viglianti, B.L.; Dewhirst, M.W. (Durham, NC)

360 Behandlung des lokal fortgeschtittenen Ösophaguskarzinoms: Es ist Zeit, unsere Untersuchungsstrategien zu ändern Ajani, J.A. (Houston, TX)

Originalarbeiten

362 Eine Pilotstudie mit sehr schwachen elektrischen Feldern von intermediärer Frequenz bei Patienten mit lokal fortgeschrittenen und/oder metastasierten Tumoren

Salzberg, M. (Basel); Kirson, E.; Palti, Y. (Haifa); Rochlitz, C. (Basel)

366 Paclitaxel in der neoadjuvanten Behandlung von Adenokarzinomen des distalen Ösophagus (AEG). Vergleich zweier Phase-II-Studien mit LangzeitFollow-Up

Bader, F.G. (Lübeck/Stockholm); Lordick, F. (Heidelberg); Fink, U.; Becker, K.; Höfler, H.; Busch, R. (München); Siewert, J.R.; Ott, K. (Heidelberg)

375 Lebermetastasenchirurgie bei gynäkologischen Tumoren - Indikation und Ergebnisse Kollmar, O.; Moussavian, M.R.; Richter, S.; Bolli, M.; Schilling, M.K. (Homburg)

380 Langzeituiberlebensanalyse beim metastasierten Melanom: Serum S100B ist ein unabhängiger prognostischer Marker und der LDH überlegen Egberts, F.; Pollex, A.; Egberts, J.H.; Kaehler, K.C.; Weichenthal, M.; Hauschild, A. (Kiel)

Kasuistiken

387 Bilaterale Metastasen eines lobulären Mammakarzinoms in den extraokulären Muskeln Kouvaris, J.R.; Gkongkou, P.V.; Papadimitriou, C.A.; Papacharalampous, X.N.; Antypas, C.E.; Balafouta, M.J.; Vlahos, L.J. (Athens)

\section{KARGER}

Fax +497614520714

E-mail Information@Karger.de

www.karger.com
(C) 2008 S. Karger GmbH, Freiburg

Artikel (Volltext) und Inhaltsverzeichnisse

sowie das vorläufige Inhaltsverzeichnis des nächsten Heftes:

www.karger.com/onk_bk.htm 


\section{Vol. 31, Issue 7, July 2008}

391 Chemorefractory Rhabdomyosarcoma Treated with Radiotherapy, Bevacizumab, Statins and Surgery and Maintenance with Bevacizumab and Chemotherapy

Lindén, O.; Greiff, L.; Wahlberg, P.; Vinge, E.; Kjellén, L. (Lund)

394 Bevacizumab plus Irinotecan-, Fluorouracil-, and Leucovorin-Based Chemotherapy with Concomitant HAART in an HIV-Positive Patient with Metastatic Colorectal Cancer

Berretta, M.; Lleshi, A.; Zanet, E.; Bearz, A.; Simonelli, C. (Aviano); Fisichella, R.; Nasti, G.; Berretta, S. (Naples); Tirelli, U. (Aviano)

Review Articles

398 Metastatic Melanoma: Scientific Rationale for Sorafenib Treatment and Clinical Results Egberts, F.; Kähler, K.C.; Livingstone, E.; Hauschild, A. (Kiel)

404 Venous Access Ports: Frequency and Management of Complications in Oncology Patients

Jordan, K.; Behlendorf, T.; Surov, A.; Kegel, T.; Maher, G.; Wolf, H.H. (Halle/Saale)

\section{Erratum}

412 PharmaForum

414 PharmaNews / PharmaTicker

416 Meetings and Conferences

418 Guidelines for Authors

397 Imprint

Forthcoming papers are listed on page 420.

\section{Band 31, Heft 7, Juli 2008}

391 Chemotherapierefraktäres Rhabdomyosarkom: Behandlung mit Radiotherapie, Bevacizumab, Statinen und Chirurgie und Erhaltungstherapie mit Bevacizumab und Chemotherapie

Lindén, O.; Greiff, L.; Wahlberg, P.; Vinge, E.; Kjellén, L. (Lund)

394 Chemotherapie mit Bevacizumab plus Irinotecan, Fluorouracil und Leucovorin mit begleitender HAART bei einem HIV-positiven Patienten mit metastasiertem Kolorektalkarzinom

Berretta, M.; Lleshi, A.; Zanet, E.; Bearz, A.; Simonelli, C. (Aviano); Fisichella, R.; Nasti, G.; Berretta, S. (Naples); Tirelli, U. (Aviano)

Übersichtsarbeiten

398 Metastasiertes Melanom: wissenschaftliche Grundlage für die Therapie mit Sorafenib und Ergebnisse klinischer Studien Egberts, F.; Kähler, K.C.; Livingstone, E.; Hauschild, A. (Kiel)

404 Venöse Portsysteme: Häufigkeit und Management von Komplikationen bei Tumorpatienten Jordan, K.; Behlendorf, T.; Surov, A.; Kegel, T.; Maher, G.; Wolf, H.H. (Halle/Saale)

403 Erratum

412 PharmaForum

414 PharmaNews / PharmaTicker

416 Tagungen und Kongresse

419 Hinweise für Autoren

397 Impressum

Einen Ausblick auf den Inhalt der kommenden Hefte finden Sie auf Seite 420.

\section{KARGER}

Fax +497614520714

E-mail Information@Karger.de

www.karger.com (c) 2008 S. Karger GmbH, Freiburg

Artikel (Volltext) und Inhaltsverzeichnisse

sowie das vorläufige Inhaltsverzeichnis des nächsten Heftes: www.karger.com/onk_bk.htm 